\title{
First-perspective spatial alignment effects from real-world exploration
}

\author{
Paul N. Wilson and Duncan A. Wilson \\ University of Hull, Hull, England \\ LAURA GRIFFITHS \\ University of Leicester, Leicester, England \\ AND \\ SARAH Fox \\ University of Hull, Hull, England
}

\begin{abstract}
When spatial knowledge is acquired from secondary-learning media, such as text, people sometimes remember a route in alignment with the first perspective or first direction of travel. However, this first-perspective alignment (FPA) effect has been found only under special circumstances from primary real-world exploration. In Experiment 1, recall of an enclosed small-scale, U-shaped route was compared following learning from a verbal description, a video recording, or real-world exploration; an FPA effect was found in all cases. In Experiments 2 and 3, exploration of physically larger real routes led to statistically significant evidence of an FPA effect when the route was enclosed, but not when cues external to the route were available. The data are discussed in relation to current theories of spatial reference frames.
\end{abstract}

A key concept in the understanding of human spatial learning and representation is the reference frame that is used to encode an environment. The spatial reference system processes the interrelationships among objects, actors, and events and includes coordinate axes and environmentally determined planes. Spatial reference frames can usefully be divided into three broad categories: egocentric, intrinsic, and allocentric. Egocentric reference frames represent locations with respect to the immediate perspective of the explorer, including retinal coordinates (Sholl \& Nolin, 1997) and body-centered axes (Bryant \& Tversky, 1999; Franklin \& Tversky, 1990). Intrinsic encoding is similar but is centered on the location and orientation of another person, animal, object, or group of objects. Allocentric reference frames are defined by global features of the environment that are external to the explorer (e.g., Easton \& Sholl, 1995; Mou, McNamara, Valiquette, \& Rump, 2004; Sholl \& Nolin, 1997).

Spatial alignment effects reflect important properties of spatial reference systems. Alignment effects are revealed when measures of spatial learning show more efficient processing from one or more recalled orientations than from others; when these effects occur, memory is said to be orientation dependent or orientation specific. An everyday example of an alignment effect is that a map that is studied in an orientation corresponding with the orientation of the environment that it represents is relatively easy to interpret (Levine, 1982) but increased difficulty is experienced as the orientation of the map rotates and becomes misaligned with the environment. A special case of misalignment is contra-alignment, in which the map is rotated by $180^{\circ}$ with respect to the environment that it represents (e.g., Levine, Jankovic, \& Palij, 1982; Rossano \& Warren, 1989).

In the laboratory, alignment effects are commonly revealed in a recall task after people have studied an environment from one or more set perspectives. Typically, participants are asked to make judgments of relative direction (JRDs) of the form, "Imagine that you are at X, facing toward Y; indicate the direction toward Z." The experimenter usually records both angular errors in the estimates and latencies to make these judgments (e.g., Levine et al., 1982; Levine, Marchon, \& Hanley, 1984; Palij, Levine, \& Kahan, 1984). Greater error suggests a less accurate internal spatial representation, and longer latencies suggest greater difficulty in adopting a particular perspective or otherwise using the spatial representation. Both measures are advisable, since it is possible to increase accuracy at a cost in judgment latencies (see Waller, Montello, Richardson, \& Hegarty, 2002).

A common example of an alignment effect is that, after an environment has been studied from a single perspective, recall is usually most efficient in imagined alignment with the studied perspective (e.g., Evans \& Pezdek, 1980;

P. N.Wilson, p.wilson@hull.ac.uk 
Levine et al., 1982; Presson, DeLange, \& Hazelrigg, 1989; Presson \& Montello, 1994; Roskos-Ewoldsen, McNamara, Shelton, \& Carr, 1998; Shelton \& McNamara, 2001a; Thorndyke \& Hayes-Roth, 1982). This effect suggests that the space is encoded in relation to the single, egocentrically determined orientation experienced during learning. However, recent research has identified a variety of other factors that can influence alignment effects. For example, in addition to learning from a single perspective, testing following exposure to multiple perspectives may reveal evidence consistent with multiple viewpointdependent representations (Shelton \& McNamara, 1997; see also Diwadkar \& McNamara, 1997). Alignment effects can also be influenced by the presence of salient external landmark information (McNamara, Rump, \& Werner, 2003). Shelton and McNamara (2001a) found that orientation-dependent performance may reflect an interaction between egocentrically experienced perspectives and regularities in the environmental structure: Where allocentric features suggest orthogonal geometric axes, these axes can influence one or more preferred orientations. Mou and McNamara (2002) found that if participants were instructed to attend to an axis defined by the intrinsic layout of cues in an array but the axis was offset from their viewing perspective, the most efficiently processed orientation was that attended to, rather than that directly seen. Similarly, asking participants to describe the layout of an object array from a perspective that is different from that actually seen can lead to efficient alignment recall from both seen and described perspectives (Shelton \& McNamara, 2004b), and manually reconstructing an array from an imagined perspective can lead to stronger alignment effects from the reconstructed perspective than from the actually seen perspective (Shelton \& McNamara, 2001b).

One alignment effect that has received relatively little direct experimental attention is the first-perspective alignment (FPA) effect. For example, Wilson, Tlauka, and Wildbur (1999) found that after participants had either read or heard a description of a tour along a right-angled, U-shaped route, errors in JRDs and latencies to make judgments were lower when the imagined orientation at test was aligned with the first part of the route description and the first direction of travel (see also Shelton \& McNamara, 2004a). This FPA effect was evident despite manipulations designed to promote multiple imagined views on the environment, such as repeatedly describing a return journey through city streets from a ground-level perspective. The effect has also been found following exploration of routes in virtual environments (Richardson, Montello, \& Hegarty, 1999; Rossano, West, Robertson, Wayne, \& Chase, 1999; Wilson \& Wildbur, 2004).

The FPA effect is important to the theory of spatial learning proposed by McNamara and his colleagues (e.g., McNamara \& Valiquette, 2004; Mou \& McNamara, 2002; Shelton \& McNamara, 2001a). According to their theory, environmental learning is always orientation dependent, with a preferred orientation being determined by both environmental structure and egocentric experience. Shelton and McNamara (2001a, Experiment 7) found evidence of the FPA effect under special circumstances: After participants had studied an array of objects on the floor of a circular enclosure that obscured surrounding features, JRDs in alignment with the first of three experienced perspectives were more accurate than those in alignment with other seen, as well as unseen, perspectives. Shelton and McNamara (2001a) suggested that under circumstances in which cues external to the array were not available to support a frame of reference, the participants adopted an egocentric reference frame in which the first viewing perspective defined a conceptual north within the array.

In the context of route learning, the theory suggests that in the absence of a defining environmental structure or other informative properties of the setting, the initial egocentric orientation or first segment of a route is the most likely aspect of experience on which to base a preferred orientation (e.g., McNamara \& Valiquette, 2004, pp. 21-22). A prediction from this account is that the absence of external spatial cues that can be used to define a spatial reference frame around a route should promote reliance on the first experienced perspective to determine a preferred orientation; however, the presence of salient allocentric cues should facilitate selection of alternative preferred orientations, attenuating the FPA effect. This prediction was tested in Experiments 2 and 3 of the present series.

Although the FPA effect is well established from secondary-learning sources (Richardson et al., 1999; Rossano et al., 1999; Shelton \& McNamara, 2004a; Wilson et al., 1999; Wilson \& Wildbur, 2004), the evidence from real-world experiments is mixed. In support of the generality of the effect, Palij et al. (1984) found evidence for an FPA effect after their participants had explored a real-world route; in this experiment, the participants were blindfolded and followed a small-scale route from beginning to end only (i.e., no return journey was experienced), and they maintained a facing orientation consistent with the first direction of travel throughout exploration. However, a number of factors preclude generalizing from the FPA findings of Shelton and McNamara (2001a) and Palij et al. Both experiments involved relationships in a smallscale space, in which the structure could be observed from a single viewpoint (typically room-sized or smaller spaces), rather than in a large-scale space, in which the structure could not be observed from a single viewpoint and knowledge of overall structure must be acquired from exploration (Kuipers, 1978). Furthermore, the procedures used in these studies were unusual (e.g., circular surround to the space or single facing direction during exploration), and it therefore remains an open question as to whether the FPA effect will be found under more typical real-world learning conditions.

Evidence inconsistent with the proposal of FPA processing from real-world learning can be found in the study by Richardson et al. (1999). They found that after participants had explored a single direction of travel along a route through the corridors on two floors of a building, either in a virtual environment (VE) simulation or in a real building, an FPA effect was apparent only in the VE case. Errors for perspectives aligned and contra-aligned 
with the first part of the route and the first direction of travel did not differ statistically in the real-world condition. However, Richardson et al. did not record latencies to make JRDs, and it is possible that alignment effects are reflected in either errors or latencies or that a trade-off between accuracy and latency masked an alignment effect (Waller et al., 2002). Without further investigation, Richardson et al.'s finding raises the possibility that features of real-world exploration - possibly, vestibular and kinesthetic feedback - lead to different alignment encoding between primary- and secondary-learning sources.

With the exception of Richardson et al.'s (1999) experiment, the FPA comparisons between learning from primary and secondary sources have relied on experiments in which the procedures and parameters, in addition to the learning media, were different. Thus, the question of whether the FPA effect occurs following learning from different media with otherwise similar parameters remains to be systematically addressed. Similar alignment effects from real-world exploration and from secondary learning would be compatible with the hypothesis that spatial information is represented in a common spatial representation system irrespective of the learning medium (e.g., Bryant, 1992; Denis, 1996; Jackendoff \& Landau, 1991; Talmy, 1983). In Experiment 1 of the present series, FPA effects following learning from secondary sources (verbal descriptions and purely visual presentation) were compared with those following real-world exploration, using similar route layouts, measures, and exposure to multiple perspectives.

If the FPA reflects a true alignment effect, in which the entire environment is encoded and recalled with respect to a preferred orientation, or conceptual north, as McNamara's (e.g., McNamara \& Valiquette, 2004; Mou \& McNamara, 2002; Shelton \& McNamara, 2001a) theory suggests, judgments should be more efficient when

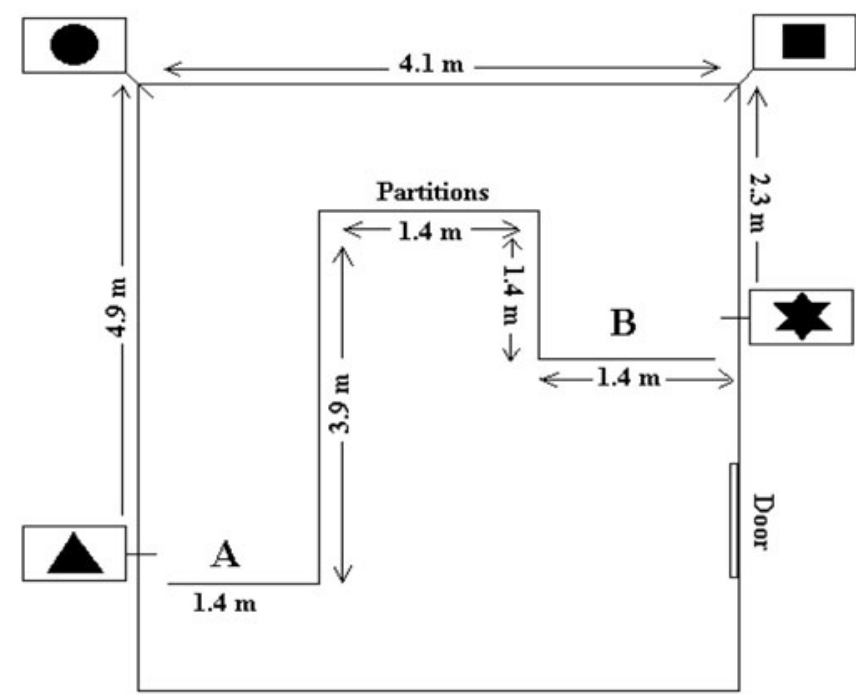

Figure 1.A plan illustration of the alleyway constructed within an experimental room used in Experiment 1. The participants first started walking from either the longest part of the route (A) or the shortest part of the route (B). aligned, rather than contra-aligned, with the first direction of travel on both the first and the last sections of the routes explored in the present series (see Figure 1). That is, if the first perspective and direction of travel is from A in Figure 1 (triangle to circle) and this direction defines the conceptual north, judgments should be more efficient from all imagined perspectives that are congruent with this orientation (e.g., triangle facing circle and star facing square) than opposite to this orientation (e.g., circle facing triangle and square facing star). However, an alternative prediction is that exploration of the return journeys in the present series could be interpreted as similar to learning a list of views. For example, with reference to Figure 1, and assuming that the tour started at A, the order of views experienced would be triangle-circle, circle-square, squarestar, star-square, square-circle, and circle-triangle. More efficient processing of the triangle-circle view than of the circle-triangle view on the first part of the route is consistent with a primacy effect in which the first view in the list is recalled more accurately than the last view in the list (e.g., Glanzer \& Cunitz, 1966). The crucial prediction from the list-learning hypothesis is that the square-star and star-square views, which are on the last section of the route but are adjacent in the middle of the list, should show little, if any, difference in recall efficiency or, possibly, a small reversal of this effect. Thus, the list-learning hypothesis predicts that the FPA should be found on the first, but not on the last, section of the route. Therefore, we collated dependent measures for the two route sections separately.

Also recorded in the present experiments were differences in the efficiency of judgments for which the correct response was in front of or behind the participants' imagined facing direction at test (i.e., whether the correct angles were less than or greater than $90^{\circ}$ ). To the extent that, in the test, the participants adopted an egocentric bodycentered frame of reference, more efficient processing would be expected for targets to the front of the imagined facing orientation than for targets behind it, due to the forward dominance of the senses (e.g., Bryant \& Tversky, 1999; Franklin \& Tversky, 1990; Sholl, 1999). In desktop VE experiments that revealed the FPA effect, using a route structure similar to that in the present experiments, Wilson and Wildbur (2004) found no evidence for the typical outcome of more efficient processing of targets to the front of the imagined test location; we wanted to see whether this outcome is generally correlated with the FPA effect.

\section{EXPERIMENT 1}

Three groups of participants were asked to walk around a small three-section alleyway, to view a video of a walk around the same alleyway, or to listen to a description of a walk around the alleyway. Since Wilson et al. (1999) and Wilson and Wildbur (2004) found evidence of an FPA effect from both verbal descriptions and VEs, using similar parameters, Experiment 1 employed routes, procedures, and measures based on these experiments. Watching a video recording of the route that was explored by the participants in the real-world condition is similar to watching 
a recorded tour through a VE (e.g., Rossano et al., 1999). On the basis of past research, we predicted that when asked to make judgments from imagined orientations that were aligned and contra-aligned with the participant's first perspective on the route and first direction of travel, lower JRD errors or lower decision latencies would be found on aligned than on contra-aligned judgments in the verbal description and video conditions. The predictions for the real-exploration condition are less clear: Because McNamara and Valiquette's (2004) account does not predict different alignment effects based on different media, an FPA effect should be observed in the real condition if it is found in the text and video conditions. However, on the basis of the findings of Richardson et al. (1999), and because additional vestibular feedback and experience of multiple return journeys might promote learning from multiple perspectives, the effect could be attenuated or absent in the real-exploration condition.

We tested only imagined perspectives that were aligned and contra-aligned with the first perspective. Although $180^{\circ}$ perspectives have sometimes been found to have a special status in alignment studies, in that processing may be more efficient from $180^{\circ}$ than from other misaligned perspectives (e.g., Roskos-Ewoldsen et al., 1998), 180 judgments are typically less efficient than aligned judgments. Furthermore, experiments in which an FPA effect has been recorded have not systematically shown less efficient processing associated with $180^{\circ}$ than with other misaligned orientations (e.g., Shelton \& McNamara, 2004a; Wilson \& Wilbur, 2004).

\section{Method}

\section{Design}

The independent variable was the method used to present the route information: The participants in the real-world condition walked through a three-alley U-shaped corridor constructed inside an experimental room. The participants in the video condition watched a video recording of a tour through these three alleys. In the verbal description condition, the participants listened to a description of a tour through the same alleys. In a subsequent test of spatial memory, all the participants were asked to make JRDs between locations while imagining themselves within the alley facing a direction that was either aligned or contra-aligned with the first direction of travel on the initial path of the route. The dependent variables were the absolute errors in JRDs and the time taken to make these judgments.

\section{Participants}

The participants were 48 undergraduate students recruited by opportunity sampling. Their mean age was 21 years (range: 20-23 years), and 35 were women. They were randomly allocated to one of three groups $(n=16)$ : The real-world group included 8 women, the video group included 14 women, and the verbal description group included 13 women.

\section{Apparatus}

For the real-world group, a three-path alleyway was constructed inside an experimental room using 1.8-m-high partitions arranged at right angles to each other (see Figure 1). Attached to the outside walls at eye level, at the ends and outside corners of the alley, were white A4 sheets of paper on which black colored shapes were presented (a triangle, circle, square, and star). The distances between the shapes were $4.9 \mathrm{~m}$ from the triangle to the circle, $4.1 \mathrm{~m}$ from the circle to the square, and $2.3 \mathrm{~m}$ from the square to the star. The alleys were portable so that the orientation of the alleyway with respect to the interior of the room and the outside environment could be moved to face in the opposite direction for half the participants.

For the video group, the experimenter filmed a walk around the three-alley pathway on three consecutive return journeys with a handheld video camera, reproducing the views experienced by the participants in the real-world group. This tour was then played back in a testing cubicle on a 36-cm (14-in.) color television.

For the verbal group, a description of the route was prepared and read aloud by the experimenter (see the Appendix). For ease of recall, the distances in the description were $8 \mathrm{~m}$ from the triangle to the circle, $4 \mathrm{~m}$ from the circle to the square, and $2 \mathrm{~m}$ from the square to the star.

Direction estimates were made using a $360^{\circ}$ protractor marked in $5^{\circ}$ intervals, consisting of a black pointing arm on a white circular dial, $20 \mathrm{~cm}$ in diameter. Response times were measured using a handheld stopwatch.

\section{Procedure}

Real-world condition. The participants were instructed to follow the route from the start to the end of the path and then back again and to immediately repeat this journey on two more occasions. On each exploration, the participants were asked to name the shapes on the wall as they encountered them. Half of the participants explored the route with the alley positioned in one orientation in the experimental room (i.e., as in Figure 1), whereas the remainder explored the same route but with the partitions moved so that the alley was facing in the opposite orientation with respect to the room and the outside environment. Across this counterbalancing of route orientation, half the participants started their exploration along the shortest part of the route first (star-square) and turned left on the first part of the journey, whereas the remainder started walking along the longest part of the route first (triangle-circle) and turned right on the first part of the journey. On completing the training phase, the participants were blindfolded and guided to a separate test room before their blindfolds were removed. They were then asked to make directional judgments between the locations of the shapes in the alleyway, as described below.

Video condition. In the video condition, the participants were asked to watch the video recording that reproduced the succession of views seen by the participants in the real-world condition on three return journeys through the alleyway. Two versions were produced to match the different start points (short or long first alley) experienced by the real-world group. Testing was conducted in the same room with the video apparatus, with the television screen made blank.

Verbal condition. The participants in the verbal description condition were tested in a quiet cubicle and were read a passage of text that described a return journey along the three-alley route, repeated on three occasions; the description included the dimensions of the alleyway and the locations of the shapes (these were the same shapes as those encountered in the video and real-world conditions). Two versions of the descriptions were prepared that corresponded with the different start points (short or long first path in the alley) experienced by the real-world and video groups. Testing was conducted in the same room.

Testing. In the test phase, all the participants were asked to make directional judgments between the shapes from memory. Judgments were made using the $360^{\circ}$ protractor, the use of which was explained to them before the experiment. Examples of aligned and contraaligned questions, respectively, are the following: "Imagine that you are standing at the triangle, and you are facing the circle which is directly ahead of you. Point towards the square" and "Imagine that you are standing at the circle, and you are facing the triangle which is directly ahead of you. Point towards the star." They were asked to respond as though their imagined test location was represented by the center of the dial and that the orientation "ahead" corresponded to $0^{\circ}$. The pointer arm was reset to $0^{\circ}$ after each judgment. The participants did not receive any feedback on their pointing accuracy.

All the participants were asked a set of eight questions, with equal numbers describing perspectives that were aligned or contra-aligned with the first part of the route. Counterbalanced across aligned and 
contra-aligned judgments was whether the test location was in front of or behind the participant's imagined location and orientation (i.e., whether the correct angle was greater or less than $90^{\circ}$ ). Half of each of these judgments asked the participants to imagine themselves on the first segment of the route, and half asked them to imagine themselves on the last segment of the route. An equal number of questions required judgments that were to the left and to the right of the participant's imagined orientation. Response latencies were recorded from the last word of the question until the participants indicated that their pointing response was complete.

\section{Results and Discussion}

Due to the large number of main effects and interactions, an alpha level of .01 was selected for all the analyses reported in this article. Orientation error scores were derived for each judgment by taking the absolute difference between the angle that the participant estimated to be the direction to the test locality and the actual angle to that locality. Due to the relatively small number of men tested in Experiments 1 and 3 and the many potential statistical interactions, and because we made no predictions related to gender, sex was not included as a factor in any analyses reported in this article.

The mean absolute aligned and contra-aligned errors for each group are presented in the upper panel of Figure 2, from which it can be seen that errors were somewhat greater in the verbal description group than in either the video or the real-world group and that aligned errors were lower than contra-aligned errors in all the groups. These data were entered into a mixed $3 \times 2 \times 2 \times 2$ ANOVA with group (real world, video, and verbal description) as the between-participants factor and alignment (aligned and contra-aligned with the first direction of travel), direction (judgments made in front of or behind the participants' imagined perspective), and route part (first or last sections of the route) as repeated measures factors. This analysis yielded a main effect of alignment $[F(1,45)=$ $\left.14.35, M S_{\mathrm{e}}=3,584, \eta_{\mathrm{p}}^{2}=.24\right]$, which reflects lower error scores when judgments required an imagined perspective aligned, rather than contra-aligned, with the first direction of travel. There was also a group $\times$ direction $\times$ route part interaction $\left[F(1,45)=5.62, M S_{\mathrm{e}}=1,371, \eta_{\mathrm{p}}^{2}=.20\right]$. An examination of the interaction did not qualify the findings of interest, and since the interaction did not involve alignment, this pattern will not be discussed further. No other main effects or interactions were statistically significant $(p \mathrm{~s}>.01)$, although the group effect approached significance $\left[F(2,42)=3.83, M S_{\mathrm{e}}=6,601, p=.03, \eta_{\mathrm{p}}^{2}=.15\right]$, suggesting that overall errors for the verbal description group were somewhat greater than those for the video and real-world groups. Notably, errors did not differ between front-facing $\left(M=61^{\circ}, S E=5.2\right)$ and back-facing $(M=$ $\left.57^{\circ}, S E=4.9\right)$ judgments, and no differences involving route section were found (see Table 1).

The mean response latency scores are presented in the lower panel of Figure 2. These data were analyzed in the same way as the error scores. This analysis yielded no statistically significant main effects or interactions ( $p \mathrm{~s}>$ $.01)$. Numerically, lower overall latency scores were apparent for aligned $(M=8.7 \mathrm{sec})$ than for contra-aligned $(M=10.4 \mathrm{sec})$ judgments. Response latencies did not dif-
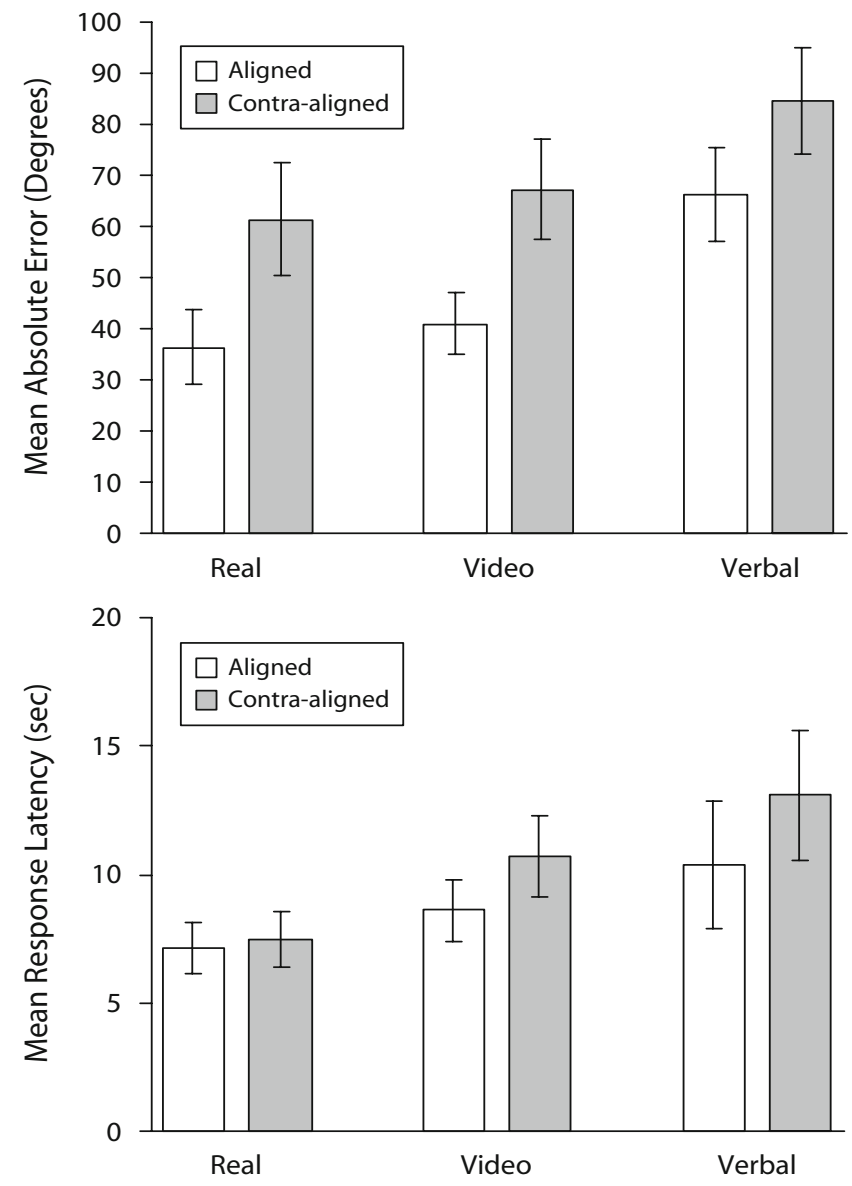

Figure 2. Mean absolute angle errors (upper panel) and mean response latencies (lower panel) for the aligned and contraaligned judgments for the group that explored the real environment (real), the group that watched a video recording of that route exploration (video), and the group that heard a description of the route (verbal), in Experiment 1. Error bars correspond to one estimated standard error above and below the mean.

fer between front-facing $(M=9.2 \mathrm{sec}, S E=0.93)$ and back-facing $(M=10.0 \mathrm{sec}, S E=1.2)$ judgments, and no differences involving route section were found (see Table 1).

In all three groups, errors in JRDs were lower when the participants imagined themselves in an orientation that was aligned, rather than contra-aligned, with the first part of the route. Latencies to make judgments did not suggest a trade-off between accuracy and decision time in any group. No differences were found in any group between alignment errors or response latencies on the first or last part of the route, and no difference was found between front- and back-facing judgments.

The data from the video group and the verbal presentation group support the experimental hypothesis in replicating the findings of Wilson and Wildbur (2004) with VEs and Wilson et al. (1999) with text, respectively. The presence of the FPA effect in the real-world group is in agreement with predictions from McNamara's (e.g., McNamara \& Valiquette, 2004; Mou \& McNamara, 2002; Shelton \& McNamara, 2001a) theory, outlined in the introduc- 
Table 1

Absolute Errors and Latencies for Aligned and Contra-Aligned (Contra) Judgments on the First and Last Sections of the Routes in Experiments 1-3

\begin{tabular}{|c|c|c|c|c|c|c|c|c|c|c|c|c|c|c|c|c|}
\hline & \multicolumn{8}{|c|}{ Error $\left({ }^{\circ}\right)$} & \multicolumn{8}{|c|}{ Latency (sec) } \\
\hline & \multicolumn{4}{|c|}{ First } & \multicolumn{4}{|c|}{ Last } & \multicolumn{4}{|c|}{ First } & \multicolumn{4}{|c|}{ Last } \\
\hline & \multicolumn{2}{|c|}{ Aligned } & \multicolumn{2}{|c|}{ Contra } & \multicolumn{2}{|c|}{ Aligned } & \multicolumn{2}{|c|}{ Contra } & \multicolumn{2}{|c|}{ Aligned } & \multicolumn{2}{|c|}{ Contra } & \multicolumn{2}{|c|}{ Aligned } & \multicolumn{2}{|c|}{ Contra } \\
\hline & $M$ & $S E$ & $M$ & $S E$ & $M$ & $S E$ & $M$ & $S E$ & $M$ & $S E$ & $M$ & $S E$ & $M$ & $S E$ & $M$ & $S E$ \\
\hline \multicolumn{17}{|l|}{ Experiment 1} \\
\hline Verbal & 70 & 9.3 & 85 & 9.9 & 63 & 9.7 & 84 & 12.1 & 9.5 & 2.1 & 12.9 & 2.5 & 11.3 & 3.2 & 13.3 & 2.7 \\
\hline Video & 35 & 5.6 & 68 & 11.9 & 47 & 9.0 & 66 & 11.5 & 7.4 & 1.0 & 9.9 & 1.5 & 9.8 & 2.0 & 11.4 & 1.9 \\
\hline Real & 39 & 9.1 & 66 & 13.6 & 34 & 9.4 & 57 & 9.5 & 7.1 & 1.4 & 7.4 & 1.1 & 6.8 & 0.8 & 7.9 & 1.3 \\
\hline Experiment 2 & 18 & 4.1 & 26 & 5.1 & 19 & 4.3 & 22 & 5.4 & 6.8 & 0.7 & 9.0 & 0.7 & 6.4 & 0.8 & 10.4 & 1.3 \\
\hline Experiment 3 & 32 & 6.9 & 42 & 7.2 & 58 & 7.8 & 70 & 13.3 & 7.2 & 0.8 & 8.9 & 0.9 & 9.4 & 1.2 & 7.1 & 0.7 \\
\hline
\end{tabular}

tion, and is contrary to predictions based on Richardson et al.'s (1999) results. This novel finding is surprising in that learning involved primary experience that included kinesthetic and vestibular feedback and the participants had direct experience of multiple perspectives as they walked along the route on three return journeys. Memories of multiple perspectives engendered by this experience might be expected to lead to equally good judgments from all perspectives and orientations.

Richardson et al. (1999) found evidence for an FPA effect after their participants had explored a VE, but not following exploration of an equivalent real-world environment. Differences between Experiment 1 of the present series and Richardson et al.'s study include experience of several return journeys in the present experiment, but only one direction of travel in Richardson et al.'s study. However, it is difficult to see how return journeys, which involve exposure to more perspectives on the environment, could promote orientation dependence in alignment with the first perspective. The other obvious difference between these studies is the scale of the environments that were explored: Experiment 1 employed a relatively small route that was constructed inside a room, but Richardson et al.'s experiment employed long corridors that traversed the length of a building. However, the theory of $\mathrm{McNa}$ mara and Valiquette (2004; see also Mou \& McNamara, 2002; Shelton \& McNamara, 2001a) does not predict a difference in alignment effects following exploration of large- and small-scale routes.

Presson et al. (1989) found that small paths (e.g., $40 \times$ $40 \mathrm{~cm}$ ) were encoded in alignment with the single viewing perspective, but this effect was reduced or eliminated in larger routes $(4 \times 4 \mathrm{~m})$. However, a lack of orientation specificity in larger environments has been difficult to replicate (Roskos-Ewoldsen et al., 1998; Sholl \& Nolin, 1997; Waller et al., 2002), and alignment effects have been clearly demonstrated in large-scale environments (e.g., McNamara et al., 2003). More directly relevant to the FPA effect, there is evidence from studies of secondary learning that increasing the dimensions of a verbally presented route with a layout similar to that in Experiment 1 leads to an FPA effect (Wilson et al., 1999); similarly, an FPA effect was found in a desktop VE that modeled streets presented in a layout similar to that in Experiment 1 (Wilson $\&$ Wildbur, 2004). This suggests that, at least for these secondary media, the FPA effect appears to be independent of the scale of the environment. Therefore, Experiment 2 concentrated on real-world learning, for which there is little direct evidence for an FPA effect. This experiment addressed the question of whether differences in scale could account for the real-world exploration differences between the results of Experiment 1 and Richardson et al.'s results by replicating the essentials of the procedure of the real-world group in Experiment 1, but employing a much larger environment.

Other noteworthy features of the results of Experiment 1 were the following. First, there was a failure to find a statistically significant difference in the magnitude of the alignment effect between the groups (i.e., no group $\times$ alignment interaction). This is surprising given that previous studies have reported a large difference between judgments that were aligned and contra-aligned with the first-perspective from text (Wilson et al., 1999), but a smaller, and sometimes statistically nonsignificant, difference from VEs (Wilson et al., 1999; Wilson \& Wildbur, 2004). The somewhat smaller alignment effect in the present verbal case appears to be due to overall poor learning in that condition. High errors on aligned trials in the verbal group suggest that the participants had difficulty memorizing the layout from the descriptions, perhaps as a consequence of the confusability of the geometric shapes, which they did not experience visually.

Second, dependent measures that involved locations in front of the imagined perspective did not differ from those that involved locations behind the imagined perspective. This is somewhat surprising if, in the test, people relied on a body-centered spatial framework in which they imagined themselves to be within the environment, because in such a case front-facing errors might be expected to be lower than back-facing errors due to the forward dominance of the senses (Bryant, Tversky, \& Franklin, 1992; Franklin \& Tversky, 1990; Sholl \& Nolin, 1997). However, the lack of a direction effect is consistent with Wilson and Wildbur's (2004) observation of no difference between front- and back-facing judgments when an FPA effect was apparent in a VE study.

\section{EXPERIMENT 2}

Experiment 2 investigated whether the size of the environment is a factor in governing the FPA effect. Par- 
ticipants explored a route that was structured similarly to that described, viewed, or explored in Experiment 1, but the route was located within the corridors of a large building more similar in scale to that explored by Richardson et al.'s (1999) participants. If evidence for an FPA effect depends on exploration of a small-scale route, no FPA effect, or an attenuated effect, should be apparent in Experiment 2. According to the theory of McNamara and colleagues (e.g., McNamara \& Valiquette, 2004; Mou \& McNamara, 2002; Shelton \& McNamara, 2001a), the scale of the environment is not predicted to influence the selection of a preferred orientation.

\section{Method}

\section{Design}

The participants were asked to learn a simple three-path route, similar to that in Experiment 1, through the corridors of a large rectangular building by direct navigation and were subsequently asked to make judgments from imagined perspectives that were aligned and contra-aligned with the first part of the route. Four variations on the route were devised (see Figure 3 ): Two routes "faced" in the same cardinal direction but had opposite directions of turn (A, B, C, $\mathrm{D}$ and $\mathrm{A} 1, \mathrm{~B} 1, \mathrm{C} 1, \mathrm{D} 1)$, whereas the other two routes "faced" in the opposite cardinal direction to the first two and also had directions of turn opposite to each other (W, X, Y, Z and W1, X1, Y1, Z1). By random allocation of equal numbers of participants to each path, with half the participants in each case walking in a clockwise direction initially and half walking in a counterclockwise direction initially, the following variables were counterbalanced: first, the orientation of the routes with respect to the external environment; second, the direction in which the participants turned on the initial exploration of the route; and, third, whether the shorter or the longer part of the route was explored first. As in Experiment 1, the dependent variables were the absolute error in the participants' JRDs and the time taken to make these judgments.

\section{Participants}

These were 24 undergraduates who participated in partial fulfillment of a course requirement. They had a mean age of 20.3 years (range: 18-28 years), and 12 were men.

\section{Apparatus}

The explored corridors formed a rectangle, $20 \mathrm{~m}$ long and $11 \mathrm{~m}$ wide, within a building on the University of Hull campus. The corridors lacked windows, so external surrounding features were not visible. Cues such as a world map, bucket, chair, painting, bookcase, and fire extinguisher served to define the target locations along the route.

\section{Procedure}

Learning phase. The participants were tested individually. Prior to exploration, they were told that they should try to remember the path that they were about to explore and, in particular, the test locations that were to be pointed out to them on the route. The experimenter then accompanied them on a walk around the designated route at their own pace and pointed out four target locations, which they were asked to name aloud. The first target location was on the wall adjacent to where the walk began; the second and third were at the corners of the building; and the last was adjacent to where the walk finished. When the path had been explored from start to end in one direction, the participant turned through $180^{\circ}$ and accompanied the experimenter back along the route to the starting point. This procedure was repeated twice. JRD testing was carried out in a single location removed from the route; during the test, equal numbers of participants sat facing "north," "south," "east," and "west" with respect to the orientation of the explored route. The test questions were similar to those in Experiment 1.

\section{Results and Discussion}

The absolute error data were collated as in Experiment 1; as can be seen in the upper panel of Figure 4, errors for judgments aligned with the first direction of travel were only slightly lower than those that were contra-aligned with the first direction of travel. A $2 \times 2 \times 2$ repeated measures ANOVA with alignment, direction (front- and back-facing), and route section (first and last sections) as factors yielded no main effects or interactions between main effects $(p \mathrm{~s}>.01)$. Front-facing judgment errors $\left(M=20^{\circ}, S E=4.2\right)$ were very similar to back-facing errors $\left(M=21^{\circ}, S E=3.0\right)$; alignment effects were similar on the first and last sections of the route (Table 1).

As is illustrated in the lower panel of Figure 4, latencies were substantially shorter for judgments that were aligned, rather than contra-aligned, with the first direction of travel. An analysis of the latency data similar to that carried out with the error data yielded a large main effect of alignment $\left[F(1,23)=36.58, M S_{\mathrm{e}}=13.01, \eta_{\mathrm{p}}^{2}=\right.$ .61]. No other main effects or interactions were statistically significant $(p \mathrm{~s}>.01)$. Front-facing latencies $(M=$ $7.4 \mathrm{sec}, S E=0.8)$ were slightly lower than back-facing latencies $(M=8.9 \mathrm{sec}, S E=0.9)$, but the difference was not statistically significant at the predefined criterion $\left[F(1,23)=5.84, M S_{\mathrm{e}}=16.83, p=.024\right]$. Alignment effects were similar on the first and last sections of the route (Table 1).

Experiment 2 showed evidence for an FPA effect in judgment latencies, although not in errors. The partici-

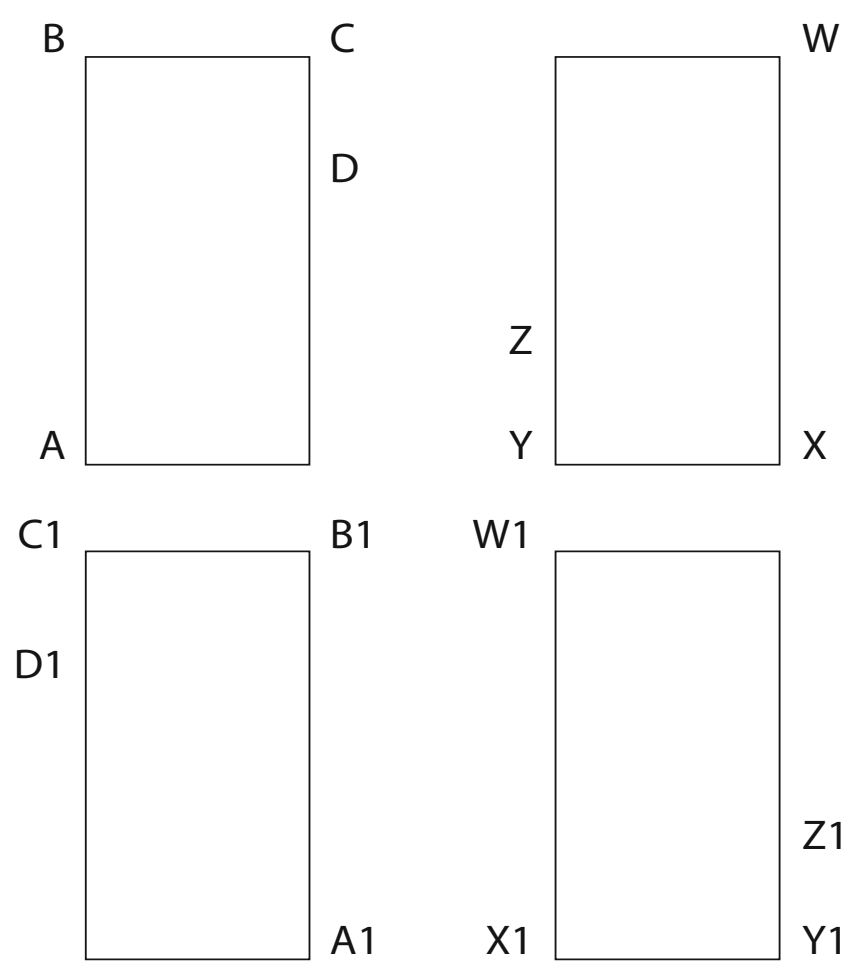

Figure 3. Plan view of the building used in Experiment 2, illustrating the four routes that formed the basis of the counterbalancing measures. 

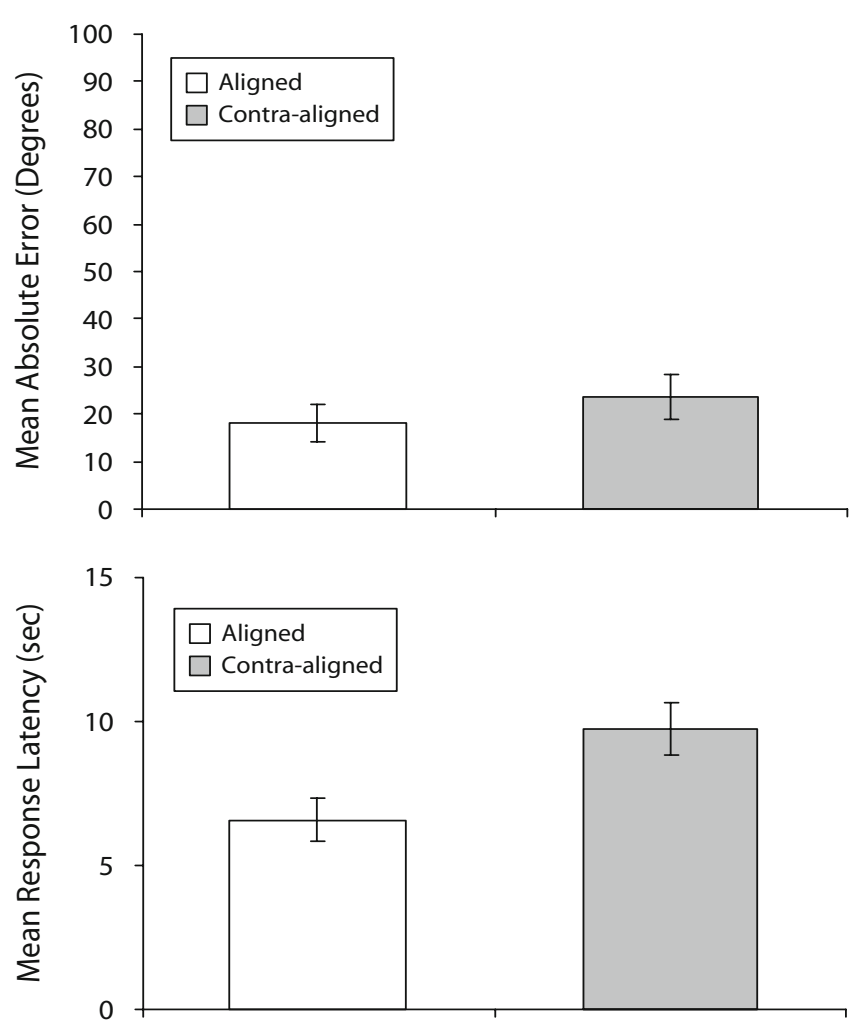

Figure 4. Mean absolute angle errors (upper panel) and mean response latencies (lower panel) for the aligned and contraaligned judgments in Experiment 2. Error bars correspond to one estimated standard error above and below the mean.

pants were faster to make judgments that were aligned with the first direction of travel. The error data in this experiment are in agreement with Richardson et al.'s (1999) finding of no FPA in errors; had latencies not been recorded, we would have concluded, as they did, that the FPA is not a feature of learning large-scale real environments. It is not clear why the FPA effect should have been apparent primarily in errors for the small-scale route in Experiment 1 but primarily in latencies for the large-scale route in Experiment 2. The data reinforce the importance of taking both dependent measures into account before deciding whether a procedure has or has not led to alignment effects. Waller et al. (2002) have found that people who do not show alignment effects in errors often show them in latencies.

Strictly, evidence of a difference in efficiency of processing between front- and back-facing judgments was not found in Experiment 2, which replicates Wilson and Wildbur's (2004) finding from VE learning and is consistent with the results of Experiment 1. However, the probability level in the analysis of the different latencies for front- and back-facing judgments $(p=.024)$, although not exceeding the predefined criterion, does weaken the case for supposing that a lack of front-back difference and the FPA are correlated.

As was outlined in the introduction, an account of the FPA effect can be suggested in terms of learning a list of views. More efficient processing of the first section of a route might be related to the well-established primacy effect in which the first items in a list are sometimes recalled more readily than the later items. According to this hypothesis, the FPA should be found for the first, but not the last, section of the route. Contrary to this hypothesis, no differences in alignment effects were found for the first and last sections of the route in Experiments 1 or 2 (see Table 1). The present data are consistent with the suggestion that, when it occurs, the FPA effect is a consequence of the entire environment's being encoded in alignment with the initial perspective.

The equivalence of the FPA effect found on the first and last sections of the route also reflects on the possibility that something about the outward journey, from the start to the point where the participants turned to begin their return journey rendered this direction of exploration more salient than the return journey and that this could account for the FPA effect. For example, although the objects were named on both the outward and the return journeys, naming the objects for the first time might be a more memorable experience. As in the case of the list-learning hypothesis, such an account would predict that on the last section of the route, the outward journey (e.g., square to star in Figure 1) should be processed more efficiently than the return journey (star to square in Figure 1). If this had been the case, judgments on the last section of the route should have shown the reverse of the FPA effect, which was clearly not the case.

\section{EXPERIMENT 3}

In Experiments 1 and 2, the route was enclosed by walls that precluded observation of surrounding allocentric features. Shelton and McNamara (2001a) reported an FPA effect only when the allocentric features of their small-scale environment were removed by surrounding the object array with a circular partition; when allocentric features were available, alignment effects were related to these features, and an FPA effect was not recorded. A prediction from these findings is that where surrounding landmarks are available, they should influence the spatial reference frame by reducing the salience of the first perspective in governing a preferred orientation. To test this prediction, in Experiment 3, participants explored a large-scale route that was located around the outside of a building, with surrounding external and distal landmarks available. On the basis of Shelton and McNamara's (2001a) findings, the presence of allocentric information was predicted to attenuate the FPA effect in comparison with Experiments 1 and 2 by providing the spatial reference system with alternative bases for establishing a preferred orientation.

\section{Method}

\section{Design}

The participants were asked to learn a simple three-path route adjacent to the outside walls of a rectangular building by direct navigation and were subsequently asked to make judgments from imagined perspectives that were aligned and contra-aligned with the first part of the route. 
Four variations on the route were devised, and counterbalancing of route experience was similar to that in Experiment 2. Four testing sites, located in different directions from the experimental route (at locations from which the building could not be seen), were used as locations to conduct the JRD tests. Therefore, the participants' orientation with respect to surrounding features during learning and testing should have had no systematic influence on their judgments. As in Experiments 1 and 2, the dependent variables were the absolute error in the participants' JRDs and the time it took to make these judgments.

\section{Participants}

These were 32 undergraduates who participated in partial fulfillment of a course requirement. They had a mean age of 20 years (range: 18-25 years), and 9 were men. Only students who confirmed that they were not familiar with the experimental building were invited to take part.

\section{Apparatus}

A rectangular building on the University of Leicester campus was selected as the site around which the paths were located. The building was $36 \mathrm{~m}$ long and $12 \mathrm{~m}$ wide. It was selected, first, as a location with no particularly salient adjacent landmark that might promote a single preferred orientation (cf. McNamara et al., 2003) and, second, as a building with which the participants were unlikely to be familiar, since it was situated on the periphery of the campus and three of the adjacent paths did not lead to other campus buildings. Features on or adjacent to the building walls (e.g., sign on the wall, a fire door, windows, a drain cover, and a post) were selected as target locations (i.e., A, B, C, etc. in Figure 3).

\section{Procedure}

The exploration procedure followed that in Experiment 2, including the same counterbalancing arrangements. The participants approached the target building from the direction that corresponded with the facing direction at the start of the route that they were to learn, and they explored a return journey on two occasions. In the test, each participant made four orientation judgments: two aligned and two contraaligned with the first path experienced on the route, with one aligned and one contra-aligned judgment front facing, and the others back facing. Whether an aligned or contra-aligned judgment was on the first or the last part of the route was counterbalanced between participants. For the test phase, the participants were led away from the target building and taken to one of four nearby locations for JRD testing.

\section{Results and Discussion}

The absolute error data were derived in the same way as those in Experiments 1 and 2 and were analyzed using a mixed $2 \times 2 \times 2$ ANOVA with route section (first and last path segments) as the between-participants factor and alignment (aligned or contra-aligned with the first part of the route) and direction (front or back facing) as withinparticipants factors. As is suggested in the upper panel of Figure 5, this analysis did not yield a main effect of alignment; however, a main effect of direction was apparent $\left[F(1,30)=8.88, M S_{\mathrm{e}}=1,638, \eta_{\mathrm{p}}^{2}=.23\right]$; the mean error for front-facing judgments was $40^{\circ}(S E=5.0)$, and the mean error for back-facing judgments was $61^{\circ}(S E=$ 7.0). The analysis also showed a main effect of route section $\left[F(1,30)=11.82, M S_{\mathrm{e}}=2,035, \eta_{\mathrm{p}}^{2}=.28\right]$, with lower errors on the first section of the route $\left(M=37^{\circ}, S E=5.0\right]$ than on the last section of the route $\left(M=64^{\circ}, S E=7.9\right)$. No interactions were found to be statistically significant.

The latency data are presented in the lower panel of Figure 5; these data were analyzed in the same way as the error data. This analysis yielded no main effect of alignment; however, a main effect of direction was apparent $\left[F(1,30)=8.67, M S_{\mathrm{e}}=18.4, \eta_{\mathrm{p}}^{2}=.22\right]$, which reflected faster judgments made to the front $(M=7.0 \mathrm{sec}, S E=$ $0.57)$ than to the back $(M=9.3 \mathrm{sec}, S E=0.76)$ of the participants' imagined orientation. No effect of route section was found (see Table 1), and no interactions were statistically significant.

No statistically significant evidence for an FPA effect was found in either dependent variable. It appears unlikely that the failure to find an FPA effect in Experiment 3 was due to a lack of power, since this experiment employed more participants than did Experiment 2 and more participants than in the real-exploration group in Experiment 1. The observed attenuation of the FPA effect, in comparison with Experiments 1 and 2, is consistent with Shelton and McNamara's (2001a) finding that the influence of the first perspective is attenuated in the presence of external landmark information.

One unexpected finding in Experiment 3 was that errors were substantially lower on the first than on the last section of the route overall (averaging across aligned and contra-aligned judgments). As in the case of the FPA effect when it occurs, the finding is surprising, because the participants had equal experience of exploring all sections of the route in both directions. This pattern of data suggests that the first part of the route was particularly memorable,
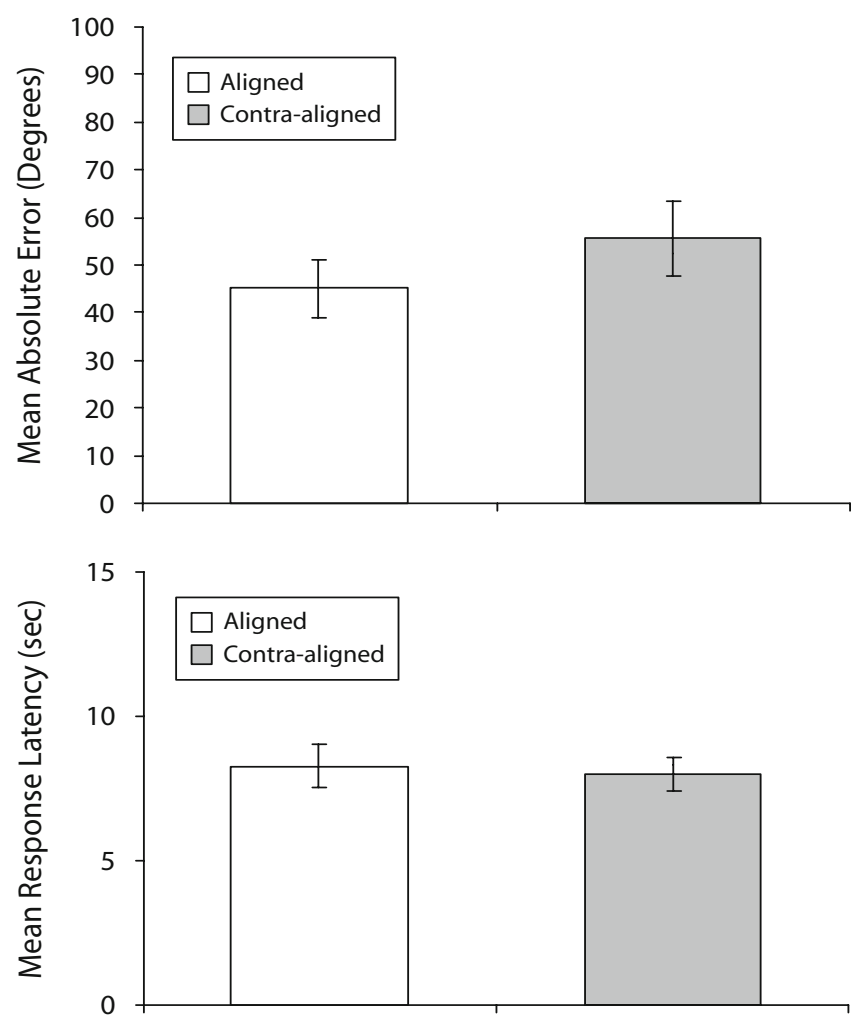

Figure 5. Mean absolute angle errors (upper panel) and mean response latencies (lower panel) for the aligned and contraaligned judgments in Experiment 3. Error bars correspond to one estimated standard error above and below the mean. 
but in a way that was rather different from that suggested by the FPA effect. Interestingly, there was no suggestion of a similar effect in the analyses of Experiment 1 or 2.

Unlike judgments following exploration of an enclosed route in Experiments 1 and 2, errors and decision times were lower when the participants were asked to judge the direction to locations that were in front of, rather than behind, their imagined orientation at test (i.e., when correct angles were less than $90^{\circ}$ ). Experiments (e.g., Bryant et al., 1992; Easton \& Sholl, 1995) have suggested that when participants adopt a body-centered egocentric perspective, they are better able to respond in front of, rather than behind, their imagined location and orientation. Therefore, this pattern of data is congruent with the hypothesis that the participants in Experiment 3 were better able to adopt a body-centered egocentric perspective than were the participants in Experiments 1 and 2, in which an FPA effect was found. It is conceivable that the availability of distal cues facilitates more accurate recall of location and orientation from previously experienced perspectives.

Interestingly, errors were substantially lower in Experiment $2\left(M=21^{\circ}\right)$ than in Experiment $3\left(M=51^{\circ}\right)$, although an FPA effect was not apparent in the error data from either experiment. Mean latencies were similar in Experiments 2 and 3 but differed in alignment effects: No FPA effect was apparent in Experiment 3, whereas the effect was present in Experiment 2. These cross-experiment observations were confirmed statistically in separate $2 \times 2$ mixed ANOVAs on the error and latency data, with experiment (Experiments 2 and 3) as the between-participants factor and alignment as the within-participants factor in both cases. In the error analysis, a main effect of experiment was found $\left[F(1,54)=19.79, M S_{\mathrm{e}}=1,214, \eta_{\mathrm{p}}^{2}=\right.$ .27], but neither the main effect of alignment nor the experiment $\times$ alignment interaction was found to be statistically significant. In the latency analysis, the main effect of experiment was not statistically significant, but a main effect of alignment was found $\left[F(1,54)=8.15, M S_{\mathrm{e}}=\right.$ $\left.6.9, \eta_{\mathrm{p}}^{2}=.13\right]$, together with an experiment $\times$ alignment interaction $\left[F(1,54)=8.15, M S_{\mathrm{e}}=6.9, \eta_{\mathrm{p}}^{2}=.18\right]$. It is not clear why errors were so much lower in Experiment 2, but one possibility is that the environment explored in that experiment did not contain the distracting surrounding features that were present in Experiment 3.

\section{GENERAL DISCUSSION}

In Experiment 1, recall was more efficient in imagined alignment with, rather than opposite to, the first experienced perspective and direction of travel, irrespective of whether learning was from direct exploration, from video presentation, or from a verbal description. A clear FPA effect was found when the participants learned an enclosed small- or large-scale route in Experiments 1 and 2, respectively, but the effect was attenuated after learning an open large-scale route in Experiment 3, in which surrounding features were available. These results are consistent with the theory of spatial reference systems proposed by McNamara and his colleagues (e.g., McNamara
\& Valiquette, 2004; Mou \& McNamara, 2002; Shelton \& McNamara, 2001a). According to this theory, spatial learning is typically orientation dependent; a principal reference vector, or conceptual north, is determined by both environmental structure and egocentric experience. Thus, factors such as the geometric properties of an enclosure, the intrinsic layout of landmarks within a defined space, or instructions to attend to particular environmental features can interact with egocentric experience to determine a preferred orientation. However, where features of the environment and procedure do not provide allocentric or intrinsic information that suggests a dominant orientation, the most likely basis for establishing a conceptual north for route learning is the initial orientation or first segment of the route (McNamara \& Valiquette, 2004). The present series provides empirical support for these proposals in experiments in which similar parameters were used in both realworld and secondary-learning procedures; the real-world experiments are the first to establish the FPA effect, using procedures that are similar to everyday exploration.

Some aspects of the data are inconsistent with other models of spatial reference frames. For example, although the results of Experiment 3 are consistent with Sholl's (1999; see also Sholl \& Nolin, 1997) model, the data from Experiments 1 and 2 are not. According to Sholl's model, two spatial reference systems interact to guide spatial judgments: first, an egocentric, body-centered frame based on the axes up-down, front-back, and leftright (see Bryant \& Tversky, 1999; Franklin \& Tversky, 1990); second, an intrinsic reference frame based on the interrelationships between landmarks in the environment. The intrinsic frame is envisaged as a network of vectors between nodes that represent objects; the relationships between objects are preserved locally with respect to each other, but not with respect to an external frame, and so the intrinsic frame is orientation free. When called upon to make directional judgments at test, the egocentric reference frame determines the participant's recalled location and orientation within the intrinsic frame. When the test location and orientation change, the egocentric frame moves to a new location within the intrinsic frame; the interrelationships between the objects in the intrinsic frame are preserved from the new perspective. Thus, the theory predicts, first, a pattern of responding that reflects body-centered egocentric encoding at all test locationsthat is, consistently more efficient front- than back-facing judgments - and second, an orientation-free intrinsic reference frame. Consistent with these assumptions, in Experiment 3, more efficient front- than back-facing judgments were found overall, and no preferred orientation was apparent. However, in Experiments 1 and 2, the presence of the FPA effect contradicts the assumption of an orientation-free intrinsic reference frame. Furthermore, no front-back direction effect was apparent in Experiment 1. Nor did Experiment 2 reveal statistically significant evidence of a front-back judgment difference; however, in Experiment 2, the slightly lower front- than back-facing latencies would have differed statistically had we adopted a less stringent alpha level. 
As the discussion above implies, it is of interest that between the experiments that did and did not find FPA effects, other differences were apparent. For example, in Experiment 3, the first segment of the route was processed more efficiently than the last, irrespective of the tested orientation with respect to the first perspective; no such asymmetry was observed where the FPA effect was found. Furthermore, where an FPA effect was evident, differences between front- and back-facing judgments were less evident, but the typical pattern of more efficient frontthan back-facing judgments was found when the FPA effect was attenuated in Experiment 3.

The finding in Experiment 3 that the first segment of the route was processed more efficiently than the last segment reinforces the suggestion of a special role for the start point of the route. Both the FPA effect and this firstsegment effect are congruent with the hypothesis that the first experienced location in a new environment forms a particularly salient anchor point (Couclelis, Golledge, Gale, \& Tobler, 1987). Couclelis et al. suggested that anchor points perform "active cognitive functions such as organizing spatial knowledge, facilitating navigational tasks, and helping estimate distances and directions etc." (p. 102). Under the relatively normal exploration conditions in Experiment 3, in which surrounding distal visual information was available, the influence of the anchor point was evident primarily in more efficient recall of views immediately adjoining the anchor point than of views on distant sections of the route. However, where visual-spatial information was restricted, as in the corridors in Experiments 1 and 2, which provided similar views at all locations, the participants' initial orientation at the anchor point determined a principal reference vector in an intrinsic object-to-object-based frame of reference.

Where evidence for this intrinsic object-to-object frame was found, greater efficiency for front- than for back-facing judgments was less evident. Although not a universal finding (e.g., Mou \& McNamara, 2002; Wilson \& Wildbur, 2004), more efficient front- than back-facing judgment is a typical outcome in the spatial-learning literature and is compatible with adoption of a body-centered frame of reference. Therefore, it remains to specify more precisely why the enclosed corridor environments in Experiments 1 and 2 should promote an object-to-object-based frame of reference, whereas the open environment in Experiment 3 apparently promoted an egocentric reference frame.

One possible account of this data pattern can be developed in terms of the distinction between lower level visual matching on a scene and the adoption of a principal reference vector when orientation judgments are made. For example, Shelton and McNamara (2004b) asked their participants to describe an array of objects from a perspective different from that actually seen; when the dependent variable was scene recognition, people were more likely to recognize the view that was actually experienced than other views. However, when the dependent variable was JRD testing, judgments congruent with the orientation that the participants had described were more efficiently processed than were those congruent with the orientation that they had seen. More relevant to the present experiments, similar effects were found for route learning after the participants had watched a VE tour through streets experienced in one direction of travel only (Shelton \& McNamara, 2004a); scene recognition was most efficient for views experienced on the individual legs of the journey, but JRD testing revealed more efficient processing for orientations in alignment with the first direction of travel - an FPA effect.

Shelton and McNamara (2004a, 2004b) suggested that these patterns of data indicate that multiple representations, or multiple components of a representation, are formed during spatial learning. A process akin to eidetic imagery stores snapshots of the environment, and these snapshots will facilitate scene recognition. However, when participants are called on to make JRDs, another process is engaged that is less dependent on explicit visual processing but represents object-to-object relations and incorporates a principal reference vector. When applied to the present experiments, JRD testing should primarily engage the object-to-object system and its principal reference vector based on the first perspective. The eidetic imagery process may have had little influence on this system in Experiments 1 and 2 because all of the images to be recalled at each location comprised similar views along corridors. Thus, the lack of a difference in front- versus back-facing judgments could have resulted from difficulty in adopting perspectives specific to each test location, with consequent reference back to the initial anchor point to determine the imagined orientation. However, in Experiment 3, in which the views at each location differed considerably due to the availability of surrounding features, the eidetic system might have played a greater role in the test phase, promoting a visually based egocentric perspective at each location that supplemented the object-to-object system. Thus, in Experiment 3, the participants may have found it easier to recall the specific views from each test location, consequently engaging more veridical, body-centered representations from the tested perspectives.

A related but more general hypothesis can be developed in the light of the wider literature on the FPA effect. In contrast to the real-world experiments presented in this article, in experiments employing VEs the FPA effect has been found after participants have explored either corridors (Richardson et al., 1999) or more open routes (Rossano et al., 1999); and where both conditions have been included in the same study, no consistent differences have been noted (Wilson \& Wildbur, 2004). Since VEs are less veridical than are real environments, a hypothesis for future investigation is that the key factor in determining the magnitude of the FPA might be the overall richness or quality of the environmental information that is available. To date, most demonstrations of the FPA effect have been found with procedures that restricted spatial information - for example, ones using text (Shelton \& McNamara, 2004a; Wilson et al., 1999), VEs (Richardson et al., 1999; Rossano et al., 1999; Wilson \& Wildbur, 2004), real-world enclosed corridors (Experiments 1 and 2 of the present series), a real-world array with obscured 
surrounding landmarks (Shelton \& McNamara, 2001a), and exploration while blindfolded (Palij et al., 1984). Perhaps the FPA effect is promoted when the environment is spatially impoverished in any way. According to this account, the attenuation of the FPA in Experiment 3 of the present series occurred because no artificial restrictions were placed on the availability of external landmark information and exploration was relatively natural. A prediction from this account is that varying the veridicality of spatial information within a single medium (e.g., text, VEs, or real environments) should systematically influence the strength of the FPA effect.

In summary, the present experiments suggest that the FPA effect is a general feature of spatial learning that reflects encoding of the entire route from the start location and orientation of exploration. These findings are compatible with predictions suggested by McNamara and Valiquette (2004; see also Mou \& McNamara, 2002; Shelton \& McNamara, 2001a). Their model proposes that egocentric experience is important in determining a reference frame and that the intrinsic features of a new environment are interpreted in terms of a principal reference vector. Congruent with their theory, the present results suggest that the first experienced location in a new environment forms a particularly salient anchor point (Couclelis et al., 1987) and that the orientation established at this anchor point determines a conceptual north. The influence of the first perspective appears to interact with environmental features, having the greatest influence where external cues are minimal.

\section{AUTHOR NOTE}

We are grateful to Michael Tlauka and Diane J. Wildbur for helpful comments on an earlier version of the manuscript. Correspondence concerning this article should be addressed to P. N. Wilson, Department of Psychology, University of Hull, Cottingham Road, Hull HU6 7RX, England (e-mail: p.wilson@hull.ac.uk).

\section{REFERENCES}

Bryant, D. J. (1992). A spatial representation system in humans. Psycoloquy, $\mathbf{3}$, space 1 .

BRYANT, D. J., \& TVERSKY, B. (1999). Mental representations of perspective and spatial relations from diagrams and models. Journal of Experimental Psychology: Learning, Memory, \& Cognition, 25, 137-156.

Bryant, D. J., TVErsky, B., \& Franklin, N. (1992). Internal and external spatial frameworks for representing described scenes. Journal of Memory \& Language, 31, 74-98.

Couclelis, H., Golledge, R. G., Gale, N., \& Tobler, W. (1987). Exploring the anchor-point hypothesis of spatial cognition. Journal of Experimental Psychology, 7, 99-122.

DENIS, M. (1996). Imagery and the description of spatial configurations. In M. de Vega, M. J. Intons-Peterson, P. N. Johnson-Laird, M. Denis, \& M. Marschark (Eds.), Models of visuospatial cognition (pp. 128197). New York: Oxford University Press.

DiwadKar, V. A., \& McNamara, T. P. (1997). Viewpoint dependence in scene recognition. Psychological Science, 8, 302-307.

EASTON, R. D., \& SHOLL, M. J. (1995). Object-array structure, frames of reference, and retrieval of spatial knowledge. Journal of Experimental Psychology: Learning, Memory, \& Cognition, 21, 483-500.

Evans, G. W., \& PezdeK, K. (1980). Cognitive mapping: Knowledge of real-world distance and location information. Journal of Experimental Psychology: Human Learning \& Memory, 6, 13-24.

FrankLin, N., \& TVERSKY, B. (1990). Searching imagined environments. Journal of Experimental Psychology: General, 199, 63-76.

Glanzer, M., \& Cunitz, A. R. (1966). Two storage mechanisms in free recall. Journal of Verbal Learning \& Verbal Behavior, 5, 351-360.
JACKendoff, R., \& LANDAU, B. (1991). Spatial language and spatial cognition. In D. J. Napoli \& J. A. Kegl (Eds.), Bridges between psychology and linguistics: A Swarthmore festschrift for Lila Gleitman (pp. 145-169). Hillsdale, NJ: Erlbaum.

KuIPERS, B. (1978). Modeling spatial knowledge. Cognitive Science, 2, 129-153.

Levine, M. (1982). You-are-here maps: Psychological considerations. Environment \& Behavior, 14, 221-237.

Levine, M., Jankovic, I. N., \& Palis, M. (1982). Principles of spatial problem solving. Journal of Experimental Psychology: General, 111, 157-175.

Levine, M., Marchon, I., \& Hanley, G. (1984). The placement and misplacement of you-are-here maps. Environment \& Behavior, 16, 139-157.

McNamara, T. P., Rump, B., \& Werner, S. (2003). Egocentric and geocentric frames of reference in memory of large-scale space. Psychonomic Bulletin \& Review, 10, 589-595.

McNamara, T. P., \& ValiquetTe, C. M. (2004). Remembering where things are. In G. L. Allen (Ed.), Human spatial memory: Remembering where (pp. 3-24). Mahwah, NJ: Erlbaum.

Mou, W., \& McNamara, T. P. (2002). Intrinsic frames of reference in spatial memory. Journal of Experimental Psychology: Learning, Memory, \& Cognition, 28, 162-170.

Mou, W., McNamara, T. P., Valiquette, C. M., \& Rump, B. (2004). Allocentric and egocentric updating of spatial memories. Journal of Experimental Psychology: Learning, Memory, \& Cognition, 30, 142157.

Palij, M., Levine, M., \& Kahan, T. (1984). The orientation of cognitive maps. Bulletin of the Psychonomic Society, 22, 105-108.

Presson, C. C., Delange, N., \& Hazelrigg, M. D. (1989). Orientation specificity in spatial memory: What makes a path different from a map of the path? Journal of Experimental Psychology: Learning, Memory, \& Cognition, 15, 887-897.

Presson, C. C., \& Montello, D. R. (1994). Updating after rotational and translational body movements: Coordinate structure of perspective space. Perception, 23, 1447-1455.

Richardson, A. E., Montello, D. R., \& Hegarty, M. (1999). Spatial knowledge acquisition from maps and from navigation in real and virtual environments. Memory \& Cognition, 27, 741-750.

Roskos-Ewoldsen, B., McNamara, T. P., Shelton, A. L., \& Carr, W. (1998). Mental representations of large and small spatial layouts are orientation dependent. Journal of Experimental Psychology: Learning, Memory, \& Cognition, 24, 215-226.

Rossano, M. J., \& Warren, D. H. (1989). Misaligned maps lead to predictable errors. Perception, 18, 215-229.

Rossano, M. J., West, S. O., Robertson, T. J., Wayne, M. C., \& Chase, R. B. (1999). The acquisition of route and survey knowledge from computer models. Journal of Environmental Psychology, 19, 101-115.

Shelton, A. L., \& McNamara, T. P. (1997). Multiple views of spatial memory. Psychonomic Bulletin \& Review, 4, 102-106.

Shelton, A. L., \& McNamara, T. P. (2001a). Systems of spatial reference in human memory. Cognitive Psychology, 43, 274-310.

Shelton, A. L., \& McNamara, T. P. (2001b). Visual memories from nonvisual experiences. Psychological Science, 12, 343-347.

Shelton, A. L., \& MCNAmara, T. P. (2004a). Orientation and perspective dependence in route and survey learning. Journal of Experimental Psychology: Learning, Memory, \& Cognition, 30, 158-170.

Shelton, A. L., \& McNamara, T. P. (2004b). Spatial memory and perspective taking. Memory \& Cognition, 32, 416-426.

Sholl, M. J. (1999). Egocentric frames of reference used for the retrieval of survey knowledge learned by map and navigation. Spatial Cognition \& Computation, 1, 475-494.

Sholl, M. J., \& Nolin, T. L. (1997). Orientation specificity in representations of place. Journal of Experimental Psychology: Learning, Memory, \& Cognition, 23, 1494-1507.

TAlmy, L. (1983). How language structures space. In H. L. Pick, Jr. \& L. P. Acredolo (Eds.), Spatial orientation: Theory, research, and application (pp. 225-282). New York: Plenum.

Thorndyke, P. W., \& Hayes-Roth, B. (1982). Differences in spatial knowledge acquired from maps and navigation. Cognitive Psychology, 14, 560-589.

Waller, D., Montello, D. R., Richardson, A. E., \& Hegarty, M. 
(2002). Orientation specificity and spatial updating of memories for layouts. Journal of Experimental Psychology: Learning, Memory, \& Cognition, 28, 1051-1063.

Wilson, P. N., Tlauka, M., \& Wildbur, D. (1999). Orientation specificity occurs in both small- and large-scale imagined routes presented as verbal descriptions. Journal of Experimental Psychology: Learning, Memory, \& Cognition, 25, 664-679.

Wilson, P. N., \& WildBur, D. J. (2004). First-perspective alignment effects in a computer-simulated environment. British Journal of Psychology, 95, 197-217.

\section{APPENDIX}

I am going to read you a passage of text that describes a walk through three short alleyways that are connected at right angles. I want you to imagine yourself walking along these alleyways, and to try to remember the position of four pictures of symbols that have been stuck on the walls at eye level. Try to envisage your movements as clearly as you can, and try to remember where the objects are located in relation to one another. You might want to close your eyes while you imagine the journey.

I will read the description three times, but will be happy to read it again if you wish. Then I will ask you to make some directional judgments between the objects on the walls from memory. To do this I will ask you to use a pointing dial. To make a directional judgment, imagine that you are at the centre of the dial, and zero degrees represents the direction that you imagine that you are facing.

Imagine that at the start of the first alley you see a picture of a triangle mounted on the wall. Beyond the triangle the path extends eight metres straight ahead. As you start walking along this path you see a picture of a circle situated at the junction ahead. You walk to the circle and make a right-hand turn. Imagine that you walk a further four metres along the next alleyway to a picture of a square at the next junction. You turn right at the square and walk two metres to a picture of a star. Imagine that at the star you turn one hundred and eighty degrees to face back towards the picture of the square. Imagine that you walk back two metres to the square and turn left. As you round the corner you can see the circle four metres ahead. Imagine that you walk back along the alley to the circle and turn left so that you are now facing the triangle. Imagine that you walk the eight metres back to the triangle.

(Manuscript received September 28, 2005;

revision accepted for publication June 6, 2006.) 